\title{
Myonectin inhibits adipogenesis in 3T3-L1 preadipocytes by regulating p38 MAPK pathway
}

\author{
Tae-Jun Park ${ }^{1,3, \#}$, Anna Park ${ }^{2,3, \#}$, Jaehoon Kim², Jeong-Yoon Kim ${ }^{1}$, Baek Soo Han ${ }^{3}$, Kyoung-Jin Oh ${ }^{3}$, Eun Woo Lee ${ }^{3}$, \\ Sang Chul Lee ${ }^{3}$, Kwang-Hee Bae ${ }^{3, *}$ E Won Kon Kim ${ }^{3, *}$ \\ ${ }^{1}$ Department of Microbiology and Molecular Biology, Chungnam National University, Daejeon 34134, ${ }^{2}$ Department of Biological Sciences, \\ Korea Advanced Institute of Science and Technology (KAIST), Daejeon 34141, ${ }^{3}$ Metabolic Regulation Research Center, Korea Research \\ Institute of Bioscience and Biotechnology (KRIBB), Daejeon 34141, Korea
}

\begin{abstract}
In current times, obesity is a major health problem closely associated with metabolic disease such as diabetes, dyslipidemia, and cardiovascular disease. The direct cause of obesity is known as an abnormal increase in fat cell size and the adipocyte pool. Hypeplasia, the increase in number of adipocytes, results from adipogenesis in which preadipocytes differentiate into mature adipocytes. Adipogenesis is regulated by local and systemic cues that alter transduction pathways and subsequent control of adipogenic transcription factors. Therefore, the regulation of adipogenesis is an important target for preventing obesity. Myonectin, a member of the CTRP family, is a type of myokine released by skeletal muscle cells. Although several studies have shown that myonectin is associated with lipid metabolism, the role of myonectin during adipogenesis is not known. Here, we demonstrate the role of myonectin during adipocyte differentiation of 3T3-L1 cells. We found that myonectin inhibits the adipogenesis of 3T3-L1 preadipocytes with a reduction in the expression of adipogenic transcription factors such as $\mathrm{C} / \mathrm{EBP} \alpha, \beta$ and PPAR $\gamma$. Furthermore, we show that myonectin has an inhibitory effect on adipogenesis through the regulation of the p38 MAPK pathway and CHOP. These findings suggest that myonectin may be a novel therapeutic target for the prevention of obesity. [BMB Reports 2021; 54(2): 124-129]
\end{abstract}

\section{INTRODUCTION}

In the majority of developed countries, due to westernized die-

*Corresponding authors. Won Kon Kim, Tel: +82-42-860-4265; Fax: +82-42-860-4149; E-mail: wkkim@kribb.re.kr; Kwang-Hee Bae, Tel: +82-42-860-4268; Fax: +82-42-860-4149; E-mail: khbae@kribb.re.kr ${ }^{\text {\#}}$ These authors contributed equally to this work.

https://doi.org/10.5483/BMBRep.2021.54.2.262

Received 26 November 2020, Revised 4 December 2020, Accepted 4 December 2020

Keywords: Adipogenesis, Myonection, p38 MAPK pathway, CHOP, Obesity tary habits and a sedentary lifestyle, obesity has grown to epidemic proportions creating a serious public health challenge. Obesity is a disease caused by a chronic imbalance of energy intake and expenditure and is regarded as a cause of the increasing occurrence of various metabolic diseases such as diabetes, hypertension, hyperlipidemia, and cardiovascular diseases (1). Obesity results from increase in mass of adipose tissue, which is driven by two main processes: 1) Hypertrophy, the increase in size of each pre-existing adipocyte and 2) hyperplasia (adipogenesis), the formation of new adipocytes from precursor cells (2). Therefore, it is important to identify the factors or drugs that can control the size and number of adipocytes as a strategy for preventing obesity. In particular, hyperplasia is a key process that determines the number of adipocytes occurring in childhood and adolescence, and thus, inhibition of this process is important for preventing obesity.

Myonectin, also known as CTRP 15, is a myokine secreted by skeletal muscle cells belonging to the $\mathrm{C} 1 \mathrm{q} / \mathrm{TNF}$-related protein (CTRP) family (3). Although the function and mechanism of myonectin has not been studied in detail, it has been reported that exercise increases its expression and amount of circulation. Moreover, expression of myonectin was altered in conditions of energy perturbation such as fasting, re-fed, and high fat diet; thus, it has been suggested that myonectin is a novel myokine in the regulation of systemic lipid homeostasis (3). Another study proposed that the plasma myonectin concentration could be a major parameter associated with metabolic syndrome and insulin resistance (4). Based on the relationship between myonectin and lipid metabolism, this study aimed to investigate a potential role for myonectin during adipogenesis.

\section{RESULTS AND DISCUSSION}

\section{Myonectin inhibits the differentiation of 3T3-L1 preadipocytes}

To investigate the effect of myonectin on 3T3-L1 cell viability, cytotoxicity was measured by quantitating the amount of ATP present in cells, which indicates the presence of metabolically active cells. When the proliferation rate of the control cells not treated with myonectin was considered to be $100 \%$, the cells 
exhibited a cell viability of more than $95 \%$ at all different concentrations of myonectin $(0.5,1$, and $2 \mu \mathrm{g} / \mathrm{ml})$ tested (Fig. 1A).

Based on these results, we treated 3T3-L1 preadipocytes with myonectin within the range $(0.05-2 \mu \mathrm{g} / \mathrm{ml})$ that did not affect cell viability during differentiation. Oil red $\mathrm{O}$ staining was performed on day 10 of differentiation when the cells were fully differentiated. In the untreated control group, the formation of lipid droplets in the cytoplasm was efficiently induced indicating proper adipocyte differentiation. On the other hand, when 3T3-L1 cells were treated with myonectin at a concentration of 0.05$2 \mu \mathrm{g} / \mathrm{ml}$, lipid droplet accumulation was reduced in a dosedependent manner (Fig. 1B).

Till now, many studies have shown that several genes and proteins directly or indirectly regulate adipogenesis $(5,6)$. Peroxisome proliferator-activated receptor $\gamma$ (PPAR $\gamma$ ) is a master regulator of adipogenic programming and is essential not only for adipogenesis but also for maintaining differentiation (7). In addition, PPAR $\gamma$ regulates the expression of enzymes such as Lipin1 and Diacylglycerol O-acyltransferase 1 (DGAT1), which promote lipid accumulation within adipocytes along with CCAAT/ Enhancer Binding Protein $\alpha(C / E B P \alpha)(8,9)$. Therefore, deletion of PPAR $\gamma$ in adipose tissues of mice blocks high fat diet-induced obesity $(10,11)$. Another adipogenic regulator $\mathrm{C} / \mathrm{EBP} \alpha$ activates downstream target genes involved in lipid formation such as PPAR $\gamma$, Lipoprotein lipase (LPL), Sterol regulatory element-binding protein 1 (SREBP1), and Fatty acid-binding protein 4 (FABP4) (11). Lipid accumulation was inhibited in the white
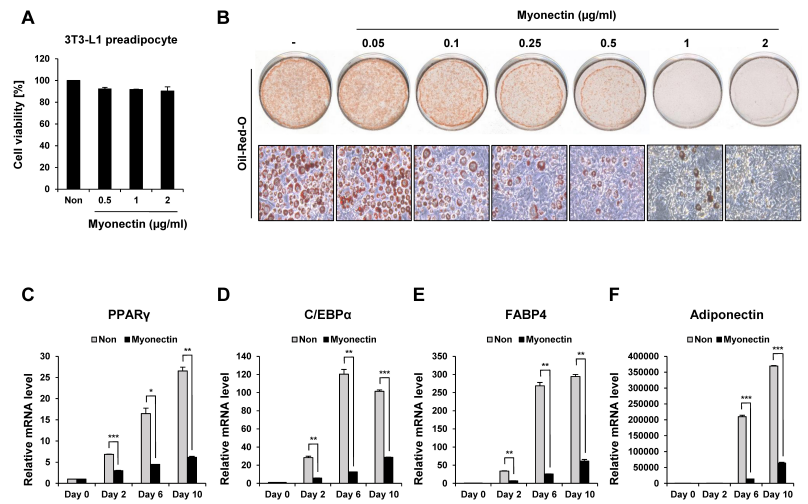

Fig. 1. Suppression of adipogenic differentiation by treatment with myonectin. (A) Effects of myonectin on cell viability. The viability of untreated control cells (Non) was defined as $100 \%$. (B) Effects of myonectin on the differentiation of 3T3-L1 cells. Differentiation of confluent 3T3-L1 cells was induced by the MDI cocktail $(0.5 \mathrm{mM}$ IBMX, $1 \mu \mathrm{M}$ Dexamethasone, and $10 \mu \mathrm{g} / \mathrm{ml}$ insulin). The 3T3-L1 cells were treated with different concentrations $(0.05-2 \mu \mathrm{g} / \mathrm{ml})$ of myonectin during differentiation. On day 10 of differentiation, the fully differentiated adipocytes were fixed and stained with oil red $\mathrm{O}$ solution for visualization of the lipid accumulation. (C-F) mRNA levels of adipogenic genes in mature 3T3-L1 adipocytes. Each gene was normalized to Rpl32. All quantitative data are the means + SD $(n=6)$. $* \mathrm{P}<0.05, * * \mathrm{P}<0.005, * * * \mathrm{P}<0.0005$ compared to untreated control cells on day 0 (Day 0, Non). adipose tissue of a mutant mouse model that lacked C/EBP $\alpha$ (12). Adiponectin is an adipokine secreted from mature adipocytes such as Leptin and Resistin and is a marker of adipocyte terminal differentiation (13). To further investigate the inhibitory effect of preadipocyte differentiation by myonectin, the expression of adipogenic transcription factors and related genes was measured at different time points (day 0, 2, 6, and 10) during differentiation. As a result, the mRNA expression levels of PPAR $\gamma$, $\mathrm{C} / \mathrm{EBP} \alpha, \mathrm{FABP} 4$, and Adiponectin in the untreated cells were increased as differentiation progressed, but the cells treated with myonectin showed a decrease at all time points (Fig. 1C-F). These results show the possibility that myonectin can inhibit adipogenesis by regulating the expression of transcription factors involved in adipocyte differentiation.

\section{Myonectin suppresses the early stage of adipogenesis}

In order to determine the specific stage of the inhibitory effect by myonectin in adipogenesis, we treated 3T3-L1 cells with myonectin at various time points (early: day 0-day 2 ; intermediate: day 2-day 6; late: day 6-day 10 ) during adipogenesis (Fig. 2A). While the groups treated with myonectin in the intermediate and late stage of adipogenesis was comparable to the control, myonectin treatment during the early stage of differentiation significantly inhibited adipogenesis by $50 \%$ compared to the control cells (Fig. 2B, C). This result showed that the inhibitory effect of myonectin in adipogenesis occurs during the initial stages of differentiation. Consistent with this result, the expression of PPAR $\gamma, \mathrm{C} / \mathrm{EBP} \alpha$, and FABP4 was

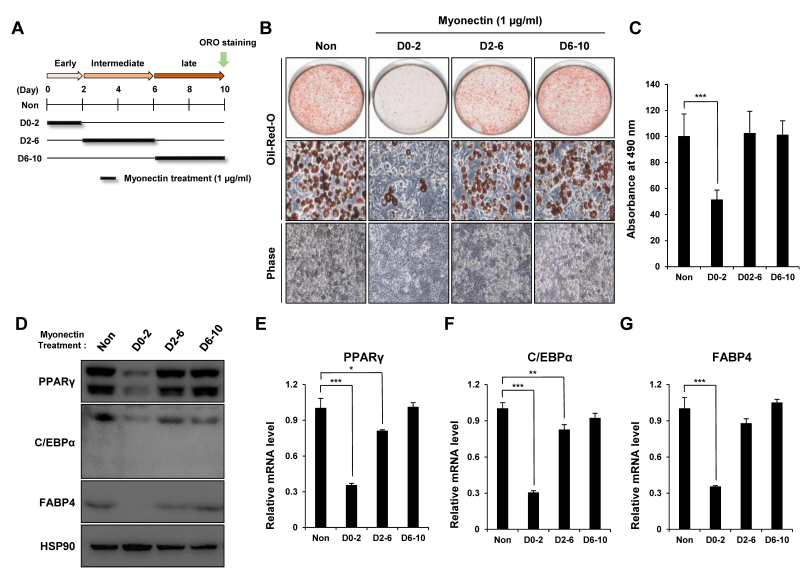

Fig. 2. Inhibitory function of myonectin involved in the early stage of adipogenesis. (A) Scheme for the preadipocyte differentiation and myonectin treatment. (B) Oil red $\mathrm{O}$ staining of lipid droplets after differentiation of the 3T3-L1 adipocytes treated with and without myonectin. (C) The levels of lipid accumulation of the 3T3-L1 mature adipocytes were quantified at $490 \mathrm{~nm}$. (D) Protein levels and (E-G) mRNA levels of the adipogenic genes in mature 3T3-L1 adipocytes treated with and without myonectin. Each gene was normalized to Rpl32. All quantitative data are the means $\pm \mathrm{SD}(\mathrm{n}=3-6)$. ${ }^{*} \mathrm{P}<0.05$ $* * P<0.005, * * * P<0.0005$ compared to the untreated control cells (Non) on day 10. 
significantly reduced in the myonectin-treated cells at the early stage of differentiation in both the mRNA and protein levels (Fig. 2D-G).

Adipogenesis of 3T3-L1 preadipocytes includes growth arrest, mitotic clonal expansion (MCE), and terminal differentiation. After growth arrest, 3T3-L1 preadipocytes are induced to differentiate by MDI cocktail containing isobutylmethylxanthine (IBMX), dexamethasone (DEX), and insulin. During the initial stage of differentiation, the adipogenic cocktail induces MCE where growth arrested preadipocytes undergo clonal expansion to roughly double in cell number followed by irreversible commitment to the adipocyte fate (6). Hyperplasia that occurs at this period is generally associated with cell proliferation signal pathways such as phosphatidylinositide 3-kinase/protein kinase B (PI3K/AKT) and mitogen-activated protein kinase/extracellular signal-regulated kinase (MAPK/ERK) pathways (14). In addition, AMP-activated protein kinase (AMPK) and $\mathrm{Wnt} / \beta$-catenin signaling are known to act at this stage $(15,16)$. These results imply that the inhibitory function of myonectin in the early stage of adipocyte differentiation may be associated with these signaling pathways mentioned above.

\section{PI3K/AKT and Wnt/ $\beta$-catenin signaling pathways are not} affected by myonectin during adipogenesis

We first identified several signaling pathways previously known to be associated with adipogenesis. In 3T3-L1 cells, activation of AKT contributes to fat cell differentiation, whereas inactivation of the PI3K/AKT pathway suppresses adipogenesis. Furthermore, the activation of AKT induced by insulin contained in the differentiation induction cocktail inactivates Forkhead box protein $\mathrm{O} 1$ (FoxO1) through phosphorylation, which is very essential in the early stage of adipocyte differentiation (17). In order to confirm the effect of myonectin on AKT, 3T3-L1 preadipocytes were treated with the MDI cocktail containing myonectin, and as a result, AKT did not show any change (Fig. 3A, B). This showed that myonectin does not affect the PI3K/AKT pathway.

As a central regulator of the intracellular energy sensor, AMPK is involved in many cellular functions regulating metabolic and biosynthetic pathways and cell proliferation and differentiation (15). In particular, activation of AMPK in adipocytes inhibits differentiation by suppressing transcription factors such as PPAR $\gamma$, C/EBP $\alpha$ and SREBP1 $(16,18)$. In our study, when 3T3-L1 preadipocytes were treated with myonectin in MDI medium, the activation of AMPK persisted for a longer time than that of the control cells (Fig. 3A, C). However, this difference in AMPK activity appeared 6 hours after the myonectin treatment, which is a relatively long time. Thus, the possibility of a secondary phenotype induced by myonectin in cells was suspected.

In the early stage of adipogenesis, it has been reported that adipogenic hormonal stimuli increase the expression of C/EBP $\beta$ and C/EBPS while inhibiting the $\mathrm{Wnt} / \beta$-catenin signaling (19, 20). In order to determine the possibility that myonectin regulates adipogenesis through $\mathrm{Wnt} / \beta$-catenin signaling, 3T3-L1 preadipo-

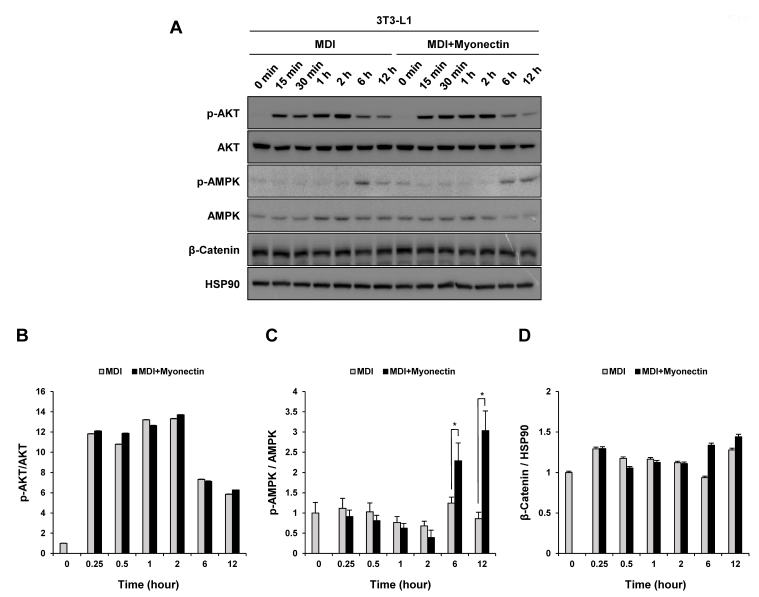

Fig. 3. Effect of myonectin on several pathways related to adipogenesis. (A) 3T3-L1 preadipocytes were induced to differentiate by the MDI cocktail with or without myonectin $(1 \mu \mathrm{g} / \mathrm{ml})$. Western blot analyses of $\mathrm{p}$-AKT, $\mathrm{p}$-AMPK and $\beta$-Catenin during the initial time of differentiation in the absence or presence of myonectin. Total AKT, total AMPK and HSP90 were presented as the controls for the normalization. (B-D) The ratios of p-AKT to total AKT, p-AMPK to total AMPK, and $\beta$-Catenin to HSP90. All quantitative data are the means $\pm \mathrm{SD}(\mathrm{n}=3)$. *P $<0.05$ compared to the myonectin-untreated control cells (MDI).

cytes were treated with myonectin in the MDI cocktail. However, there was still no change in $\beta$-catenin caused by myonectin (Fig. 3A, D).

\section{Myonectin inhibits adipogenesis through the p38 MAPK pathway}

Another pathway known as the adipogenesis regulatory pathway is the MAPK/ERK pathway. The MAPK/ERK pathway consists of ERKs, c-Jun N-terminal kinases (JNKs), and p38 MAPK belonging to the MAPK family (21). In general, ERK is activated by mitogens such as serum or growth factors; thus, this pathway acts as a key regulator of the cell cycle and cell proliferation. p38 and JNK are known to be involved in the pathway of cell stress or apoptosis in response to various stress stimuli such as UV or cytokines $(22,23)$.

Several roles of the MAPK/ERK pathway have also been reported in adipocytes. In the case of ERK, in the early stage of adipogenesis, it activates C/EBP $\beta$. Additionally, it has been reported that it is associated with obesity by contributing to the insulin resistance state through the inhibition of insulin signaling (24, 25). JNK is also known to be related to the development of obesity with its involvement in insulin signaling (26). However, we did not observe any significant changes in ERK or JNK activation by myonectin treatment (Fig. 4A).

Interestingly, however, the activity of p38 was increased and more sustained compared to the control group in the myonectin-treated cells (Fig. 4A, B). The role of p38 MAPK signaling during adipogenesis is remains unclear with both pro- and anti- 

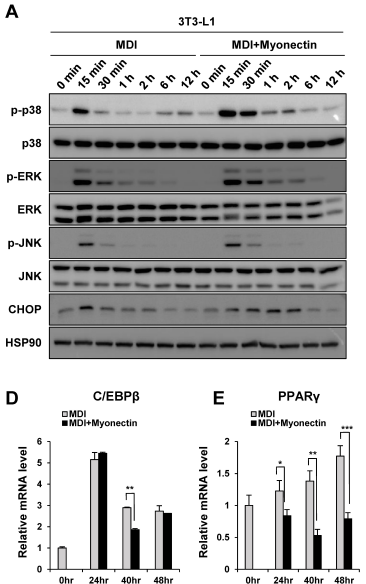

B

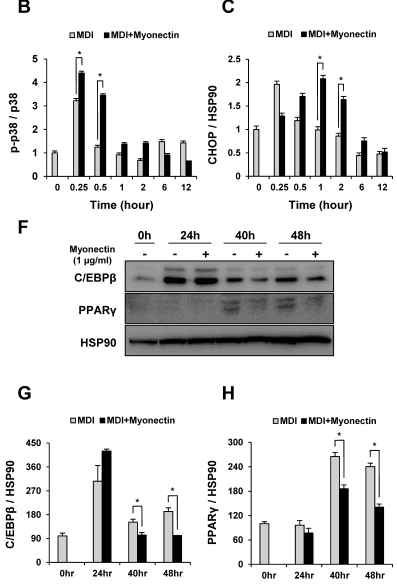

Fig. 4. Inhibitory function of myonectin on adipogenesis through the p38 MAPK pathway. (A) 3T3-L1 preadipocytes were induced to differentiate by the MDI cocktail with or without myonectin $(1 \mu \mathrm{g} /$ $\mathrm{ml}$ ). Western blot analyses of p-p38, p-ERK, p-JNK and CHOP during the initial time of differentiation in the absence or presence of myonectin. Total p38, total ERK and total JNK and HSP90 were presented as controls for normalization. ( $B, C$ ) The ratios of p-p38 to total p38 and CHOP to HSP90. (D, E) mRNA levels and (F) protein levels of $\mathrm{C} / \mathrm{EBP} \beta$ and PPAR $\gamma$. Each mRNA expression was normalized to Rpl32. $(\mathrm{G}, \mathrm{H})$ The ratios of $\mathrm{C} / \mathrm{EBP} \beta$ and PPAR $\gamma$ to HSP90. All quantitative data are the means $\pm \mathrm{SD}(\mathrm{n}=3)$. ${ }^{*} \mathrm{P}<0.05, * * \mathrm{P}<0.005$ compared to the myonectin-untreated control cells (MDI, $0 \mathrm{~min}$ ).

adipogenic functions being reported. Several studies have shown that p38 promotes adipogenic differentiation (27-29). In contrast, many other studies have suggested that p38 inhibits adipogenesis (30-36). This is probably because the p38 MAPK pathway regulates several different functions in the cell by interacting in combination with various cellular environments and other factors. Among them, there is an interesting result showing that activated p38 prevents differentiation of 3T3-L1 preadipocytes by activating the $\mathrm{C} / \mathrm{EBP}$ homologous protein (CHOP), which acts as a dominant-negative regulator of C/EBPs (36). Based on this, as a result of confirming the change in $\mathrm{CHOP}$ by myonectin treatment, $\mathrm{CHOP}$ was increased in the myonectin-treated cells (Fig. 4A, C). Moreover, this increase of $\mathrm{CHOP}$ led to a reduction of $\mathrm{C} / \mathrm{EBP} \beta$ and PPAR $\gamma$, which are the downstream targets, in both of their mRNA and protein levels (Fig. 4D-H). These results show the possibility that myonectin inhibits adipogenesis by sequentially activating p38 and CHOP in the early stage of differentiation of 3T3-L1 preadipocytes.

It remains possible that there are other factors, apart from $\mathrm{CHOP}$, that might be related to the inhibitory effect on adipogenesis by $\mathrm{p} 38$. Therefore, further studies are required for deeper understanding of the mechanism. Nevertheless, our findings have open the possibility of myonectin being a potential novel therapeutic target to prevent obesity by showing a distinct inhibitory effect on the differentiation of 3T3-L1 cells.

\section{MATERIALS AND METHODS}

\section{Culture and differentiation of 3T3-L1 preadipocytes}

The white preadipocyte cell line, 3T3-L1 cells, was purchased from ATCC. The cells were cultured in high glucose Dulbecco's Modified Eagle Medium (DMEM) containing 10\% bovine calf serum (BCS, Gibco-Invitrogen, USA) and $1 \%$ antibiotic solution at $37^{\circ} \mathrm{C}$ in a humidified atmosphere with $5 \% \mathrm{CO}_{2}$. The $3 \mathrm{~T} 3-\mathrm{L} 1$ cells were induced to differentiate into mature adipocytes as described in our previous reports $(37,38)$. The recombinant myonectin was purchased from Aviscera Bioscience (\#00393-06100, USA).

\section{Cell viability assay}

Cell viability was determined by cellular ATP levels, which indicate the presence of metabolically active cells using the CellTiter-Glo reagent (G9243, Promega) according to the manufacturer's instructions.

\section{Oil red O staining}

Lipid droplets in mature adipocytes were stained using the oil red O protocol, as described previously (39). For the quantification analysis, the stained oil red $\mathrm{O}$ dye was eluted with absolute isopropanol, and the absorbance was measured at 490 $\mathrm{nm}$ with a VersaMax microplate reader (Molecular devices, USA).

\section{RT-qPCR analysis}

Total RNA was extracted from cultured cells using TRIzol reagent (Invitrogen) according to the manufacturer's instructions (40). And first-strand complementary DNA (cDNA) was synthesized from $2 \mu \mathrm{g}$ of total RNA using the M-MLV reverse transcriptase, random primer and Rnasin RNase inhibitor (Promega, USA) according to manufacturer's instructions. Amplified cDNA was analyzed by qPCR using a SYBR green PCR kit and primers. Each gene expression level was normalized by the 605 ribosomal protein L32 (Rpl32). The primer sequences were as follows: mouse PPAR $\gamma$ (forward, 5'- CCCTGGCAAAGCATTTGTAT -3', reverse, 5'-CAAGAATACCAAAGTGCGATCAA-3'); mouse C/EBP $\alpha$ (forward, 5'-GGACAAGAACAGCAACGAGT-3', reverse, 5'-GC AGTTGCCATGGCCTTGA-3'); mouse C/EBP $\beta$ (forward, 5'-CA AGCTGAGCGACGAGTACA-3', reverse, 5'-CAGCTGCTCCACC TTCTTCT-3'); mouse FABP4 (forward, 5'-GTCACCATCCGGTC AGAGAG-3', reverse, 5'-TCGACTTTCCATCCCACTTC-3', and mouse Adiponectin (forward, $5^{\prime}$-ACACCGAGATTTCCTTCAAA CTG-3', reverse, 5'-CCATCTAGGGTTATGATGCTCTTC-3').

\section{Immunoblotting}

Homogenized cells were ice-cold lysed by ice-cold RIPA buffer [50 mM Tris-Cl (pH 7.6), $150 \mathrm{mM} \mathrm{NaCl}, 1 \mathrm{mM}$ EDTA, 0.5\% Sodium deoxycholate, $1 \%$ Triton X-100, $50 \mathrm{mM} \beta$-glycerophosphate, 50 $\mathrm{mM} \mathrm{NaF}$, and $1 \mathrm{mM} \mathrm{Na} \mathrm{VO}_{4}$ ] containing protease inhibitor (Roche, Switzerland). After centrifugation at 13,000 rpm for 15 min, supernatants were transferred to a new tube. Protein con- 
centrations were measured using the Bradford assay (Bio-Rad, USA). Western blot analysis was performed using standard protocols (41). The following primary antibodies were used: PPAR $\gamma$ (2435s, CST), C/EBP $\alpha$ (2295, CST), C/EBP $\beta$ (sc7962, Santa Cruz), FABP4 (2120s, CST), Akt (9272s, CST), p-Akt (9271s, CST), AMPK $\alpha$ (2532s, CST), p-AMPK $\alpha$ (2535s, CST), $\beta$-Catenin (06-734, Sigma), p38 (9212s, CST), p-p38 (9215s, CST), ERK (9102s, CST), p-ERK (9106s, CST), JNK (9252s, CST), p-JNK (9251s, CST), CHOP (2895s, CST), and HSP90 (sc-13119, Santa Cruz). Specific antibody signals were detected by horseradish peroxidase-conjugated secondary antimouse or anti-rabbit IgG antibody (Santa Cruz) and detected with an enhanced chemiluminescence system (Fusion Solo $\mathrm{S}$, Vilber Lourmat, France). The relative amounts of each protein band were quantified with the ImageJ software.

\section{Statistical analysis}

Data were presented as the mean \pm standard deviation (SD). The statistical significance of the comparisons was determined using the Student's two-tailed t-test. A P-value less than 0.05 was considered statistically significant.

\section{ACKNOWLEDGEMENTS}

This work was supported by grants from the KRIBB and the Research Program (Grant numbers: 2019R1A2C1006035 and 2020R1A2C2007111) of the Korea National Research Foundation.

\section{CONFLICTS OF INTEREST}

The authors have no conflicting interests.

\section{REFERENCES}

1. Spiegelman BM and Flier JS (2001) Obesity and the regulation of energy balance. Cell 104, 531-543

2. Arner P and Spalding KL (2010) Fat cell turnover in humans. Biochem Biophys Res Commun 396, 101-104

3. Seldin MM, Peterson JM, Byerly MS, Wei Z and Wong GW (2012) Myonectin (CTRP15), a novel myokine that links skeletal muscle to systemic lipid homeostasis. J Biol Chem 287, 11968-11980

4. Mi Q, Li Y, Wang M et al (2019) Circulating C1q/TNFrelated protein isoform 15 is a marker for the presence of metabolic syndrome. Diabetes Metab Res Rev 35, e3085

5. Farmer SR (2006) Transcriptional control of adipocyte formation. Cell Metab 4, 263-273

6. Chang E and Kim CY (2019) Natural products and obesity: a focus on the regulation of mitotic clonal expansion during Adipogenesis. Molecules 24, 1157

7. Rosen ED and MacDougald OA (2006) Adipocyte differentiation from the inside out. Nat Rev Mol Cell Biol 7, 885-896

8. Koh YK, Lee MY, Kim JW et al (2008) Lipin1 is a key factor for the maturation and maintenance of adipocytes in the regulatory network with CCAAT/enhancer-binding protein alpha and peroxisome proliferator-activated receptor gamma 2. J Biol Chem 283, 34896-34906

9. Takeuchi K and Reue K (2009) Biochemistry, physiology, and genetics of GPAT, AGPAT, and lipin enzymes in triglyceride synthesis. Am J Physiol Endocrinol Metab 296, E11951209

10. Jones JR, Barrick C, Kim KA et al (2005) Deletion of PPAR gamma in adipose tissues of mice protects against high fat diet-induced obesity and insulin resistance. Proc Natl Acad Sci U S A 102, 6207-6212

11. Tang QQ, Zhang JW and Daniel Lane M (2004) Sequential gene promoter interactions of C/EBPbeta, C/EBPalpha, and PPARgamma during adipogenesis. Biochem Biophys Res Commun 319, 235-239

12. Darlington GJ, Wang N and Hanson RW (1995) C/EBP alpha: a critical regulator of genes governing integrative metabolic processes. Curr Opin Genet Dev 5, 565-570

13. Koerner A, Kratzsch J and Kiess W (2005) Adipocytokines: leptin-the classical, resistin-the controversical, adiponectin-the promising, and more to come. Best Pract Res Clin Endocrinol Metab 19, 525-546

14. Kim H and Sakamoto K (2012) (-)-Epigallocatechin gallate suppresses adipocyte differentiation through the MEK/ERK and PI3K/Akt pathways. Cell Biol Int 36, 147-153

15. Hardie DG (2011) AMP-activated protein kinase: an energy sensor that regulates all aspects of cell function. Genes Dev 25, 1895-1908

16. Huang B, Yuan HD, Kim DY, Quan HY and Chung SH (2011) Cinnamaldehyde prevents adipocyte differentiation and adipogenesis via regulation of peroxisome proliferatoractivated receptor-gamma (PPARgamma) and AMP-activated protein kinase (AMPK) pathways. J Agric Food Chem 59, 3666-3673

17. Nakae J, Kitamura T, Kitamura Y, Biggs WH 3rd, Arden KC and Accili D (2003) The forkhead transcription factor Foxo1 regulates adipocyte differentiation. Dev Cell 4, 119129

18. Chen YY, Lee MH, Hsu CC, Wei CL and Tsai YC (2012) Methyl cinnamate inhibits adipocyte differentiation via activation of the CaMKK2-AMPK pathway in 3T3-L1 preadipocytes. J Agric Food Chem 60, 955-963

19. Bennett CN, Ross SE, Longo KA et al (2002) Regulation of Wnt signaling during adipogenesis. J Biol Chem 277, 3099831004

20. Kim WK, Choi HR, Park SG, Ko Y, Bae KH and Lee SC (2012) Myostatin inhibits brown adipocyte differentiation via regulation of Smad3-mediated beta-catenin stabilization. Int J Biochem Cell Biol 44, 327-334

21. Bost F, Aouadi M, Caron L and Binetruy B (2005) The role of MAPKs in adipocyte differentiation and obesity. Biochimie 87, 51-56

22. Pearson G, Robinson F, Beers Gibson T et al (2001) Mitogenactivated protein (MAP) kinase pathways: regulation and physiological functions. Endocr Rev 22, 153-183

23. Johnson GL and Lapadat R (2002) Mitogen-activated protein kinase pathways mediated by ERK, JNK, and p38 protein kinases. Science 298, 1911-1912

24. Hu J, Roy SK, Shapiro PS et al (2001) ERK1 and ERK2 activate CCAAAT/enhancer-binding protein-beta-dependent gene transcription in response to interferon-gamma. J Biol 
Chem 276, 287-297

25. Zick Y (2001) Insulin resistance: a phosphorylation-based uncoupling of insulin signaling. Trends Cell Biol 11, 437441

26. Hirosumi J, Tuncman G, Chang L et al (2002) A central role for JNK in obesity and insulin resistance. Nature 420, 333-336

27. Engelman JA, Lisanti MP and Scherer PE (1998) Specific inhibitors of p38 mitogen-activated protein kinase block 3T3-L1 adipogenesis. J Biol Chem 273, 32111-32120

28. Hata K, Nishimura R, Ikeda F et al (2003) Differential roles of Smad1 and p38 kinase in regulation of peroxisome proliferator-activating receptor gamma during bone morphogenetic protein 2-induced adipogenesis. Mol Biol Cell 14, 545-555

29. Engelman JA, Berg AH, Lewis RY, Lin A, Lisanti MP and Scherer PE (1999) Constitutively active mitogen-activated protein kinase kinase 6 (MKK6) or salicylate induces spontaneous 3T3-L1 adipogenesis. J Biol Chem 274, 35630-35638

30. Ho IC, Kim JH, Rooney JW, Spiegelman BM and Glimcher $\mathrm{LH}$ (1998) A potential role for the nuclear factor of activated T cells family of transcriptional regulatory proteins in adipogenesis. Proc Natl Acad Sci U S A 95, 15537-15541

31. Patel NG, Holder JC, Smith SA, Kumar S and Eggo MC (2003) Differential regulation of lipogenesis and leptin production by independent signaling pathways and rosiglitazone during human adipocyte differentiation. Diabetes 52, 43-50

32. Aouadi $M$, Laurent $K$, Prot $M$, Le Marchand-Brustel $Y$, Binetruy $B$ and Bost $F$ (2006) Inhibition of p38MAPK increases adipogenesis from embryonic to adult stages. Diabetes $55,281-289$

33. Yang TT, Xiong Q, Enslen $\mathrm{H}$, Davis RJ and Chow CW (2002) Phosphorylation of NFATc4 by p38 mitogen-acti- vated protein kinases. Mol Cell Biol 22, 3892-3904

34. Tamura K, Sudo T, Senftleben U, Dadak AM, Johnson R and Karin M (2000) Requirement for p38alpha in erythropoietin expression: a role for stress kinases in erythropoiesis. Cell 102, 221-231

35. Wang XZ and Ron D (1996) Stress-induced phosphorylation and activation of the transcription factor CHOP (GADD 153) by p38 MAP Kinase. Science 272, 1347-1349

36. Batchvarova N, Wang XZ and Ron D (1995) Inhibition of adipogenesis by the stress-induced protein CHOP (Gadd153). EMBO J 14, 4654-4661

37. Kim WK, Lee CY, Kang MS et al (2008) Effects of leptin on lipid metabolism and gene expression of differentiationassociated growth factors and transcription factors during differentiation and maturation of 3T3-L1 preadipocytes. Endocr J 55, 827-837

38. Kim WK, Jung H, Kim DH et al (2009) Regulation of adipogenic differentiation by LAR tyrosine phosphatase in human mesenchymal stem cells and 3T3-L1 preadipocytes. J Cell Sci 122, 4160-4167

39. Kim EY, Kim WK, Kang HJ et al (2012) Acetylation of malate dehydrogenase 1 promotes adipogenic differentiation via activating its enzymatic activity. J Lipid Res 53, 1864-1876

40. Hossain M, Imran KM, Rahman MS, Yoon D, Marimuthu V, Kim YS (2020) Sinapic acid induces the expression of thermogenic signature genes and lipolysis through activation of PKAVCREB signaling in brown adipocytes. BMB Rep 53, 142-147

41. Nguyen MT, Min KH \& Lee W (2020) MiR-183-5p induced by saturated fatty acids regulates the myogenic differentiation by directly targeting FHL1 in C2C12 myoblasts. BMB Rep $53,605-610$ 